\title{
Extensional viscosity for polymer melts measured in the filament stretching rheometer
}

Bach, Anders; Rasmussen, Henrik K.; Hassager, Ole

Published in:

Journal of Rheology

Link to article, DOI:

$10.1122 / 1.1545072$

Publication date:

2003

Document Version

Publisher's PDF, also known as Version of record

Link back to DTU Orbit

Citation (APA):

Bach, A., Rasmussen, H. K., \& Hassager, O. (2003). Extensional viscosity for polymer melts measured in the filament stretching rheometer. Journal of Rheology, 47(2), 429-441. https://doi.org/10.1122/1.1545072

\section{General rights}

Copyright and moral rights for the publications made accessible in the public portal are retained by the authors and/or other copyright owners and it is a condition of accessing publications that users recognise and abide by the legal requirements associated with these rights.

- Users may download and print one copy of any publication from the public portal for the purpose of private study or research.

- You may not further distribute the material or use it for any profit-making activity or commercial gain

- You may freely distribute the URL identifying the publication in the public portal

If you believe that this document breaches copyright please contact us providing details, and we will remove access to the work immediately and investigate your claim 


\title{
Extensional viscosity for polymer melts measured in the filament stretching rheometer
}

\author{
Anders Bach \\ The Danish Polymer Centre, Department of Chemical Engineering, Technical \\ University of Denmark, DK-2800 Kgs. Lyngby, Denmark \\ Henrik Koblitz Rasmussen \\ The Danish Polymer Centre, Department of Manufacturing Engineering and \\ Management, Technical University of Denmark, DK-2800 Kgs. Lyngby, Denmark \\ Ole Hassager ${ }^{a}$ \\ The Danish Polymer Centre, Department of Chemical Engineering, Technical \\ University of Denmark, DK-2800 Kgs. Lyngby, Denmark
}

(Received 1 July 2002; final revision received 9 December 2002)

\section{Synopsis}

A new filament stretching rheometer has been constructed to measure the elongational viscosity of polymer melts at high temperatures. Two polymer melts, a LDPE and a LLDPE, were investigated with this rheometer. A constant elongational rate has been obtained by an iterative application of the Orr-Sridhar method for specification of the end-plate movement. Agreement has been found with linear viscoelastic measurements performed in shear. Hencky strains up to about 6 have been reached. Steady values of the viscosity have been sustained in some cases for about two Hencky strain units. (C) 2003 The Society of Rheology. [DOI: 10.1122/1.1545072]

\section{INTRODUCTION}

The two most commonly used techniques for the measurement of elongational viscosity of polymer melts are the rheometric scientific RME and the Münstedt tensile rheometer. The prototype RME rheometer was constructed by Meissner and Hostettler (1994) in Zürich. The polymer filament undergoing investigation is held between four clamps while the elongational motion is applied by belts mounted on the clamps. Recently a number of RME instruments have been used in a round robin test to characterize a given LLDPE sample [Schulze et al. (2001)]. In the alternative Münstedt (1979) design, the sample is held between two clamps or glued to two end plates and subsequently stretched by moving one clamp away from the other. Common to both designs is a large initial aspect ratio of the polymer sample.

In 1990, Matta and Tytus introduced a novel device intended to measure elongational viscosity of both Newtonian and viscoelastic liquids. A small quantity of liquid is inserted between two coaxial cylinders that are vertically oriented one above the other. The upper

\footnotetext{
a) Author to whom all correspondence should be addressed; electronic mail: oh@kt.dtu.dk
} 

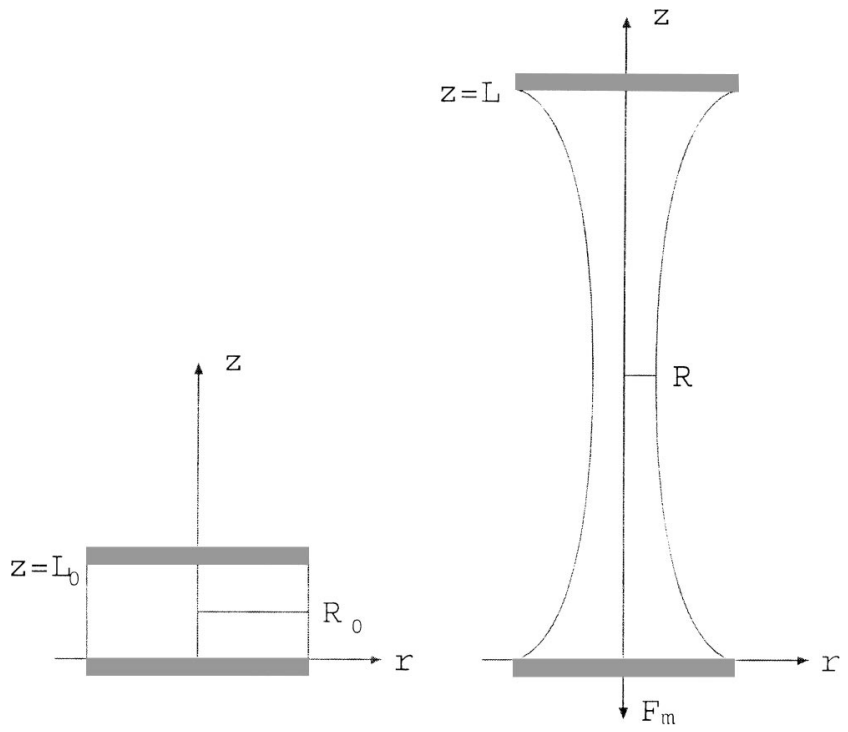

FIG. 1. Geometry of the filament in a cylindrical coordinate system $(r, z)$ with initial plate separation and midfilament radius $\left(L_{0}, R_{0}\right)$ and the corresponding quantities $(L, R)$ at time $t$.

cylinder is held fixed, while the lower cylinder, initially at rest, is allowed to fall by gravity and thereby do the stretching of the material. Sridhar et al. (1991) improved the design by moving the nonstationary plate with a motor, thereby creating the first controlled filament stretching rheometer (FSR). Since then, a number of FSRs have been constructed by several groups [Spiegelberg et al. (1996); Kröger et al. (1992); Berg et al. (1994); Solomon and Muller (1996); Van Nieuwkoop and Von Czernicki (1996); Verhoef et al. (1999)] as reviewed recently by McKinley and Sridhar (2002). Common to these designs is a low or moderate aspect ratio of the initial polymer sample. Designed initially for dilute polymer solutions, the technique is now perfected enough to measure on concentrated solutions at room temperature [Bhattacharjee et al. (2002)]. Several instruments are currently at a state where very reproducible results can be obtained by different groups working with the same fluids [Anna et al. (2001)].

Despite the reliability of the FSR technique, the method has, to our knowledge, not been successfully applied to polymer melts at high temperatures. This may be due to problems with temperature gradients, reliable measurement of deformation, or end-plate slip. We have addressed these potential problems and report here elongational viscosities of polymer melts measured with a FSR, comparing our results with measurements done on RME rheometers.

\section{THE FILAMENT STRETCHING RHEOMETER}

In the basic FSR a cylindrical filament is placed between two parallel plates (see Fig. 1 ). At times $t \geqslant 0$, the plates are separated at such a rate that the mid-radius, $R$, decreases exponentially in time. Then the strain rate $\dot{\epsilon}$ defined as

$$
\dot{\epsilon}=-\frac{2}{R} \frac{d R}{d t}
$$


is constant in time. In this way, the Lagrangian fluid element in the middle of the filament is subjected to an ideal uni-axial extension.

From measurements of the radius $R(t)$ in the symmetry plane of the filament we define the transient elongational viscosity [Bird et al. (1987)] as follows:

$$
\bar{\eta}^{+}(t)=\frac{F}{\pi R^{2} \dot{\epsilon}} .
$$

Here $F(t)$ is the force transmitted across the symmetry plane of the filament and $\dot{\epsilon}$ is the average value of the measured strain rate defined in Eq. (1). $F$ is related to the force $F_{m}$ measured at the bottom plate by a force balance [Szabo (1997)] which we take in the absence of surface tension and inertia to be

$$
F=m_{1} g+F_{m} .
$$

Here $g$ is the acceleration of gravity and $m_{1}$ is the mass of the lower half of the filament, which can be measured by forcing the filament to break after the end of an experiment. This measurement then serves to calibrate the weight cell. Provided gravity and inertia are negligible, the small distance between the end plates combined with the no slip conditions will ensure that a filament which is initially cylindrical will be thinnest in the middle of the filament. Thus, the low initial sample aspect ratio typical for FSRs is in fact important to ensure that measurement is always placed at the thinnest place in the filament.

Shaping the filament perfectly is particularly important when measuring at high strain rates. The material behaves very elastic and the reverse squeeze flow in the beginning of the experiment is not sufficient to ensure that the thinnest place is in the middle of the filament. The procedure used to obtain a constant value of $\dot{\epsilon}$ at the center of the filament is explained in Sec. IV.

\section{APPARATUS}

The rheometer is constructed as a vertical filament stretching rheometer where the bottom plate is stationary and the upper plate is pulled. The design is based on a previous built rheometer [Bach et al. (2003)]. Around this we have built a thermostated environment referred to as the oven in the following. The oven is constructed in copper for good heat conduction in the oven walls and therefore small overall temperature gradients. A heat shield surrounding the oven lowers the heat conduction in the radial direction as shown in Fig. 2. In this way we ensure high heat conduction along the oven walls and low heat conduction perpendicular to the oven walls. The temperature in the oven is regulated by six temperature measurements and proportional integral differential controllers. The heat flow is generated by eight heat elements, two more than temperature measurements because of symmetry in the oven design. At the bottom of the oven, preheated nitrogen flows into the oven chamber. Besides ensuring an inert atmosphere in the oven where the filament is placed, the nitrogen flow also helps in smearing out temperature gradients in the oven. Slits are placed in the sides of the oven and the heat shield in the axial direction of the oven. The slits are covered with glass, making it possible for a Keyence LS7500 laser to measure the diameter of the filament at a given place during an experiment. The laser is capable of measuring filament diameters in the range of $0.3-30 \mathrm{~mm}$ with an accuracy of $\pm 10 \mu \mathrm{m}$, independent of small variations in intensity that might originate from the glass covering the slits in the oven wall. The laser not only measures the diameter of the filament, but also records video images of the area 


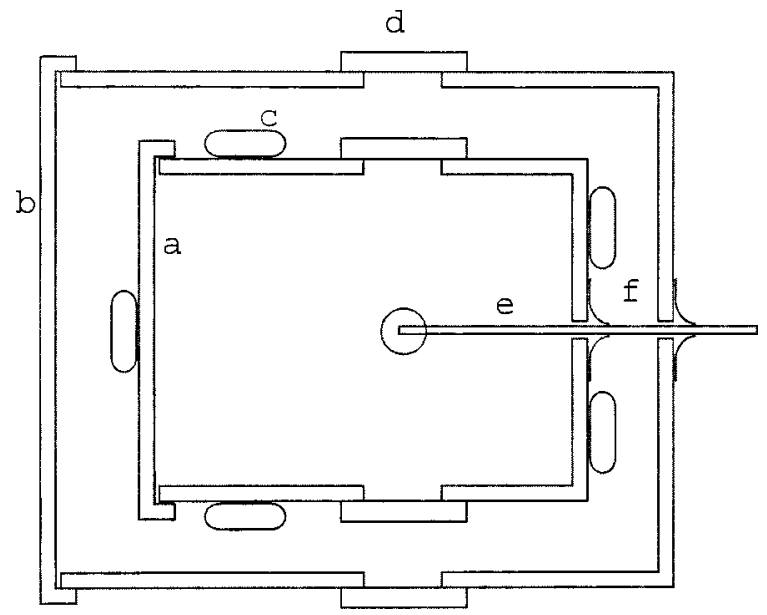

FIG. 2. Cross section of the oven. (a) Inner lid. (b) Outer lid. (c) Heat element. (d) Glass cover. (e) Arm connecting upper end plate and outer sled. (f) Rubber lips.

around the place where the diameter is measured. This gives a good assessment of the initial shape of the sample and the eventual necking of the filament in the final stages of an experiment.

At the bottom of the oven the fixed end plate is mounted on a weight cell placed outside the oven. The weight cell is a HBM Q11 $500 \mathrm{~g}$ for high force measurements and a HBM Q11 $10 \mathrm{~g}$ for small forces. Resolution of the $500 \mathrm{~g}$ cell was $0.2 \mathrm{~g}(0.2 \mathrm{cN})$ when calibrated and $0.01 \mathrm{~g}(0.01 \mathrm{cN})$ for $10 \mathrm{~g}$ weight cell. The more sensitive weight cell not only has a lower maximum force, but also a higher compliance. A thin polyimide rod connects the bottom end plate to the weight cell through a small hole in the bottom of the oven. Since the weight cell is placed outside the oven, the cell did not have to be calibrated at different measuring temperatures, as is the case for the RME [Bastian (2001)]. The laser and the upper plate are mounted on sleds that move in the axial direction. They are driven by the same motor shaft, but the toothed wheels driving the sleds have a diameter ratio of 1:2 making the laser move at half the speed of the upper plate. In this way, the laser is always placed half way between the nominal position of the end plates. However, a compliance of the weight cell will perturb the effective position of the lower plate. In this way the choice of weight cell is a compromise between accuracy of force measurement and accuracy of radius measurement during start-up.

The upper end plate is connected to the sled outside the oven by an arm designed to minimize heat flow out of the oven. A polyimide rod placed between the end plate and the arm further reduces the heat flow away from the upper plate and thus from the filament. The slit where the arm grips into the oven is covered by long "lips"sheets overlaying each other as illustrated in Fig. 2.

The motor driving the two sleds is a step motor (StebonFDT603) with a Parker OEM650 step drive and an AT6200 controller. A 1:25 planetary gear is mounted on the axis, enabling the upper sled to move at a maximum velocity of $0.5 \mathrm{~m} / \mathrm{s}$. For faster experiment a more powerful motor is used-a Parker Zeta drive $\zeta 240$ and a RS240C motor. The steel reinforced timing belts transfers momentum to the sleds and allows the position of the upper sled to be determined by the angle of rotation of the axis of the motor. An encoder, mounted on the motor is used to measure this angle whereby the position of the sled is determined. 
TABLE I. Characterization of polymer melts, data from Bastian (2001).

\begin{tabular}{|c|c|c|c|c|}
\hline Polymer melt & LLDPE & \multicolumn{3}{|c|}{ LDPE } \\
\hline Product & Affinity PL1880 & \multicolumn{3}{|c|}{ Lupolen $1810 \mathrm{H}$} \\
\hline Producer & DOW & \multicolumn{3}{|c|}{ BASF } \\
\hline$M_{w}(\mathrm{~g} / \mathrm{mol})$ & 116400 & \multicolumn{3}{|c|}{188000} \\
\hline$M_{n}(\mathrm{~g} / \mathrm{mol})$ & 55100 & \multicolumn{3}{|c|}{16600} \\
\hline$M_{w} / M_{n}$ & 2.1 & \multicolumn{3}{|c|}{11.3} \\
\hline$T_{m}\left({ }^{\circ} \mathrm{C}\right)$ & 102.5 & \multicolumn{3}{|c|}{110} \\
\hline$T\left({ }^{\circ} \mathrm{C}\right)$ & 130 & \multicolumn{3}{|c|}{150} \\
\hline Relaxation & $g_{i}(\mathrm{~Pa})$ & $\tau_{i}(\mathrm{~s})$ & $g_{i}(\mathrm{~Pa})$ & $\tau_{i}(\mathrm{~s})$ \\
\hline \multirow[t]{8}{*}{ spectrum } & $4.83 \times 10^{+5}$ & $1.00 \times 10^{-3}$ & $2.00 \times 10^{+5}$ & $9.22 \times 10^{-4}$ \\
\hline & $2.80 \times 10^{+5}$ & $7.20 \times 10^{-3}$ & $6.20 \times 10^{+4}$ & $1.13 \times 10^{-2}$ \\
\hline & $1.27 \times 10^{+5}$ & $5.18 \times 10^{-2}$ & $2.97 \times 10^{+4}$ & $1.04 \times 10^{-1}$ \\
\hline & $2.97 \times 10^{+4}$ & $3.73 \times 10^{-1}$ & $1.08 \times 10^{+4}$ & $1.06 \times 10^{+0}$ \\
\hline & $6.97 \times 10^{+3}$ & $2.68 \times 10^{+0}$ & $2.85 \times 10^{+3}$ & $8.57 \times 10^{+0}$ \\
\hline & $1.79 \times 10^{+3}$ & $1.93 \times 10^{+1}$ & $6.05 \times 10^{+2}$ & $5.40 \times 10^{+1}$ \\
\hline & $1.36 \times 10^{+2}$ & $1.39 \times 10^{+2}$ & $5.66 \times 10^{+1}$ & $3.31 \times 10^{+2}$ \\
\hline & $1.46 \times 10^{+0}$ & $1.00 \times 10^{+3}$ & & \\
\hline$\eta_{0}=\Sigma_{i} g_{i} \tau_{i}$ & $9.37 \times 10^{4} \mathrm{~Pa} \mathrm{~s}$ & & $9.13 \times 10^{4} \mathrm{~Pa} \mathrm{~s}$ & \\
\hline
\end{tabular}

\section{EXPERIMENTAL PROCEDURE}

We have investigated a linear low density polyethylene, LLDPE (DOW chemical company), and a branched polyethylene, LDPE (BASF). The properties of the melts are listed in Table I. The LDPE and the LLDPE have previously been characterized at Institut für Kunststofftechnologie, Universität Stuttgart [Bastian (2001)]. Further, the LLDPE had been the subject of a round-robin test [Schulze et al. (2001)]. Details of the RME rheometer design and experimental procedure are given in Bastian (2001). Experiments were performed at $130^{\circ} \mathrm{C}$ for the LLDPE and $150{ }^{\circ} \mathrm{C}$ for the LDPE. We note that other measurements on the LDPE exist in the literature [Wagner et al. (2000)], but these measurements were on another grade. Our measurements and the measurements from Bastian (2001) are all from the same grade.

The polymers, supplied in pellets, were pressed into cylindrical test specimens with $R_{0}=4.5 \mathrm{~mm}$ and two different lengths, $L_{0}=2.5$ and $4.5 \mathrm{~mm}$ giving aspect ratios of $\Lambda_{0}=L_{0} / R_{0}=0.56$ and 1.0.

One of the anticipated problems with measuring elongational viscosity for melts in the FSR was the possibility of the filament to peel off the end plates. For experiments reported in this work, however, end-plate slip was not a problem if proper care was taken when applying the filament to the end plates. We used end plates made of stainless steel formed as cylinders with a diameter of $9 \mathrm{~mm}$ and a height of $7 \mathrm{~mm}$. At the end of the cylinders a rod with thread was placed for tightening the end plate to the setup. In order to ensure good contact between the plates and the melt, a specimen was initially placed on top of one end plate inside the oven. After about $1 \mathrm{~h}$ at experimental temperature, good contact was obtained but at the same time the specimen would have sagged a little. This end plate was now placed in the setup as the upper plate, with the filament hanging down. The oven was heated to the test temperature and the upper end plate was lowered until the filament touched the lower end plate. The filament was allowed to sag back into its original cylindrical shape and at the same time adhere to the bottom plate. Although a perfect cylindrical shape could not be obtained, the shape of the filament could be shaped to become very close to cylindrical in this way. 

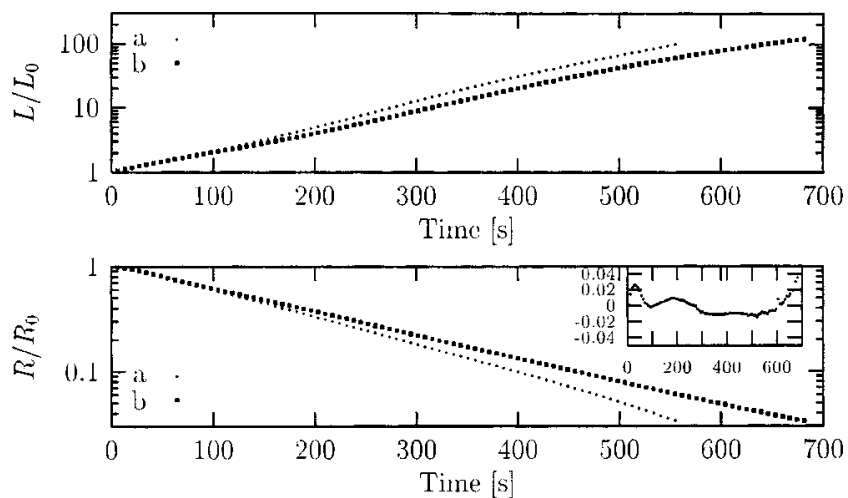

FIG. 3. Specified plate separation (top) and measured midfilament radius (bottom) as functions of time. Points "a" represent the initial trial while "b" represents the final separation profile after four iterations. Also inserted (bottom upper right corner) is the relative deviation from the desired exponential functional form of the radius.

Because of the rigid end plates, the relation between the extent of separation of the end plates and the minimum radius of the filament at the middle is not trivial. We use an approach inspired by the "master curve" approach suggested by Orr and Sridhar (1999) to generate a relationship between plate separation $L(t)$ and filament radius $R(t)$. The relation is obtained by measuring $R(t)$ in an experiment with a given specified $L(t)$. The relationship is then expressed in terms of a function $f$ such that

$$
\ln \frac{L}{L_{0}}=f\left(-2 \ln \frac{R}{R_{0}}\right),
$$

where $f(x)$ is a low order polynomial. In fact ideal elongational flow would correspond to $f(x)=x$. To obtain the desired plate separation the relation is then inverted by specifying $R(t)=R_{0} \exp (-\dot{\epsilon} t / 2)$ and calculating the resulting $L(t)$ from Eq. (4).

Orr and Sridhar found the function $f$ to be nearly independent of strain rate for their polymer solution. For the polymer melts used here we have found it necessary to apply the Orr-Sridhar procedure iteratively. Usually, at least three experiments had to be performed before a satisfying development in radius is achieved. In Fig. 3 (top) we show the plate separation as function of time. Points " $a$ " correspond to the initial experiment while points " $b$ " is the profile in the fourth and final iteration. The corresponding radii are shown in Fig. 3 (bottom). The relative deviation from the desired exponential decay of the radius is shown in the upper righthand corner of Fig. 3 (bottom). The deviation shows a maximum of about $3 \%$ in the initial stage of the experiment but decays to $\pm 1 \%$ for $\dot{\epsilon} t \in[1,6]$.

The use of nitrogen rather than a neutrally buoyant fluid sets a lower limit on the extension rate at which sagging of the filament can be neglected. A relevant measure of the magnitude of gravitational forces relative to the viscous forces is the ratio of the Reynolds number to the Froude number, $L \rho g / \eta_{0} \dot{\epsilon}$, where $\rho$ is the density of the polymer melt [Anna et al. (2001)]. From this we estimate that sagging of the polymer melts used in this investigation can be neglected at $\dot{\epsilon} \geqslant 10^{-2}$ s.

Finally, the validity of neglecting surface tension is checked by calculation of the surface elasticity number [Spiegelberg and McKinley (1996); Rasmussen and Hassager (2001)] which is an estimate of the ratio of stresses due to surface tension to the elastic modulus. Values of the order $10^{-5}$ for the two polymer melts indicate that surface tension can be neglected. 


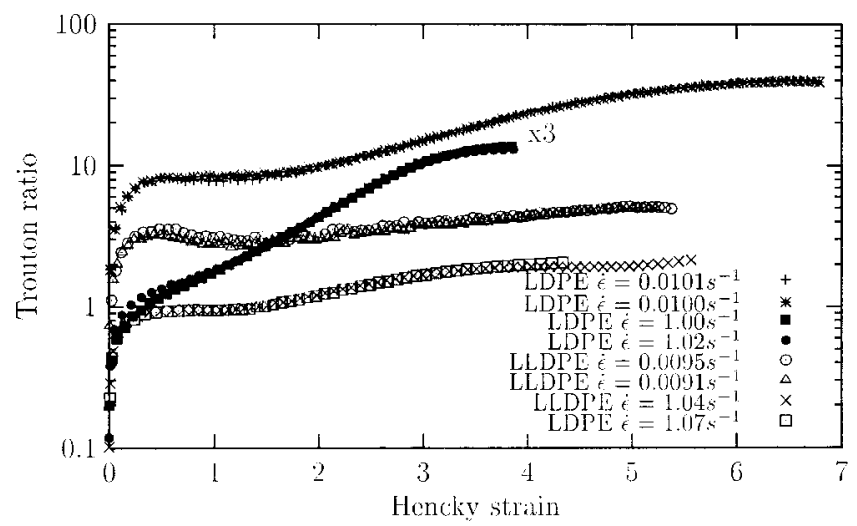

FIG. 4. Illustration of reproducibility of experiments. The Trouton ratio is plotted as function of Hencky strain for $\operatorname{LDPE}\left(150^{\circ} \mathrm{C}\right)$ and $\operatorname{LLDPE}\left(130^{\circ} \mathrm{C}\right)$ at $\dot{\epsilon}=0.01 \mathrm{~s}^{-1}$ and $\dot{\epsilon}=1.0 \mathrm{~s}^{-1}$. Two experiments are shown for each strain rate and for each melt. All measurements were performed with $\Lambda_{0}=0.56$. The measurements at $\dot{\epsilon}=0.01 \mathrm{~s}^{-1}$ have been multiplied by 3 .

\section{RESULTS}

To test the validity of the rheometer, we examine several qualities of the rheometer, that should exist. First, we report on the reproducibility of the measurements for the LLDPE and the LDPE melt at low and high strain rates. Second, the data obtained must represent material functions independent of design parameters such as the initial aspect ratio of the test specimen. In particular we compare measurements in the FSR at small strains with predictions based on the linear viscoelastic properties measured in small angle oscillations in shear. Finally, we compare the nonlinear response of the two melts with measurements done on two RME instruments and on the prototype Meissner and Hostettler instrument.

All elongational viscosities have been calculated using Eqs. (2) and (3). Since the force in the filament is measured with a weight cell, a zero load has to be established. The zero was set by measuring the load on the cell after the filament had broken up corresponding to $F=0$ in Eq. (3). This way, the effect of gravity pulling on the lower part of the filament was also calibrated away.

Results are presented as Trouton ratio plotted as function of Hencky strain. We choose these formulations to reduce the effect of deviations in strain rate in the experiments when comparing with RME data. We define the Trouton ratio as

$$
\operatorname{Tr}=\frac{\bar{\eta}^{+}}{\eta_{0}},
$$

where $\bar{\eta}^{+}$is the measured elongational viscosity and $\eta_{0}$ is the zero shear rate viscosity given in Table I. The Hencky strain $\epsilon$ in an experiment is in the FSR calculated as

$$
\epsilon(t)=-2 \ln \frac{R(t)}{R_{0}}
$$

$R(t)$ and $R_{0}$ are the radius at time $t$ and $t=0$, respectively. The strain rates in the experiments were estimated by fitting radius versus time as an exponential function. 


\section{A. Reproducibility}

In Fig. 4 we plot the Trouton ratio of the two melts at $\dot{\epsilon}=0.01 \mathrm{~s}^{-1}$ and at $\dot{\epsilon}=1.0 \mathrm{~s}^{-1}$. Two experiments are showed for each strain rate. The strain rates are reported in the caption with three digits in order to label the experiments. The experiments with $\dot{\epsilon}=1.00 \mathrm{~s}^{-1}$ and $\dot{\epsilon}=1.02 \mathrm{~s}^{-1}$ show a difference of about $15 \%$ due to a small difference in the initial acceleration of the bottom plate. Apart from this region the reproducibility is better than $5 \%$.

For LDPE at $\dot{\epsilon}=1.0 \mathrm{~s}^{-1}$ the transition from increasing stress to a saturation in stress occurs rapidly. This rapid saturation of stress in the plane of measurement combined with the stored elastic energy in the planes closer to the end plates makes the filament unstable [McKinley and Hassager (1999)]. This situation requires a rapid control of the motion of the end plates. When it is no longer possible to move the end plates sufficiently rapidly to control the radius, the filament breaks rapidly. For these materials it is more difficult to control the radius in the fast experiments than in the slow experiments as discussed in Kolte et al. (1997).

\section{B. Comparison with linear viscoelasticity}

The theory of linear viscoelasticity may be used to predict the transient elongational viscosity at small strains from the relaxation modulus. However, at small strains there is an extra force contribution from the shear components in the deformation field during startup. The extra shear component originates from the no slip condition at the rigid end plates. For Newtonian fluids this reverse squeeze flow problem can be modeled analytically so the effect of the shear may eliminated by a correction factor [Spiegelberg et al. (1996)]:

$$
\bar{\eta}_{\text {corr }}^{+}=\bar{\eta}_{\text {uncorr }}^{+}\left[1+\frac{1}{3 \Lambda_{0}^{2}} \exp \left(-\frac{7 \epsilon}{3}\right)\right]^{-1}
$$

Note that for $\Lambda_{0}>0.5$ the effect is negligible after about one strain unit.

In this work, however, we have chosen not to use this correction in the presentation of the experiments. First of all, we prefer to present raw data. Moreover, although the correction works well for LLDPE, it seems less appropriate for the highly nonlinear LDPE, for which the shearing of the sample is located very close to the end plates. To compare with linear viscoelastic predictions we have found it more appropriate to use a high initial aspect ratio. Thus, at an initial aspect ratio of $\Lambda_{0}=1.0$ it is possible to measure linear viscoelastic properties of LDPE without any corrections since the center part of the filament deforms as a perfect cylinder. In Fig. 5, we show the transient elongational viscosity of the LDPE at strain rate $\dot{\epsilon}=0.1 \mathrm{~s}^{-1}$. The figure includes three experiments performed at the same strain rate, all with $\Lambda_{0}=1.0$. The experiments show good agreement with the predictions from linear viscoelasticity.

\section{Effect of initial aspect ratio}

Also shown in Fig. 5 is one experiment performed with $\Lambda_{0}=0.56$. First of all, it may be seen, that the raw data give values higher than the prediction from linear viscoelasticity in an ideal elongation. Indeed the shift is consistent with Eq. (7) for Hencky strains less than unity. The important point, however, is that the influence of the initial shearing is limited to Hencky strain less than unity. The measured transient elongational viscosity at Hencky strains larger than unity do not depend on $\Lambda_{0}$. 


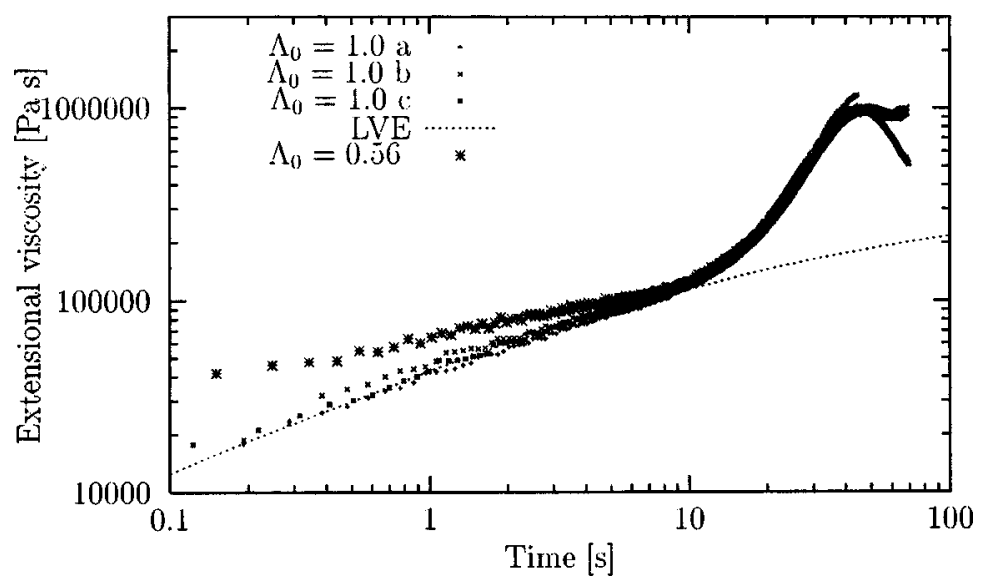

FIG. 5. Transient elongational viscosity of the LDPE melt at $150{ }^{\circ} \mathrm{C}$ and $\dot{\epsilon}=0.1 \mathrm{~s}^{-1}$. Experiments a, b, and c are performed at aspect ratio $\Lambda_{0}=L_{0} / R_{0}=1.0$. Also shown is the linear viscoelastic prediction (-).

Optimum control of the experiment is in fact obtained with a low initial aspect ratio. The reason for this is that with a low $\Lambda_{0}$ the location of the neck is predictable, namely in the middle of the filament where the laser sheet is located. For this reason we prefer to use a low initial aspect ratio. We present therefore in the following raw FSR data with a rather low initial aspect ratio with the caveat that Trouton ratios for Hencky strains less than unity should be predicted from linear viscoelasticity.

By contrast with a high $\Lambda_{0}$ the location of the neck typically occurs closer to one of the end plates. Specifically for the experiments with $\Lambda_{0}=1.0$ we observed a phenomenon that we call asymmetric break. In these situations the neck always occurs at the transition region between where the filament deforms as an ideal cylinder and the shearing region close to the end plates. We examined this observation by weighing the amount of fluid sample at each end plate after break up. In some experiments we found $72 \%$ of the filament on the upper plate and $28 \%$ on the bottom. In other experiments run at the same conditions, the distribution was reversed, $72 \%$ was now on the lower plate. Running the same experiment with lower aspect ratio $\left(\Lambda_{0}=0.56\right)$ showed an equal distribution of fluid on the upper and lower plates after break, indicating that at lower aspect ratios, the filament breaks in the middle.

\section{Comparison with RME measurements}

The ability of the FSR to measure linear viscoelastic properties of a polymer melt, is a good validation of both the technique and the instrumentation of the rheometer at small strains. However, at Hencky strains larger than about 1, polymer melts show marked deviations from linearity. Hence, an appreciation of the instrument for large Hencky strains has to be obtained from comparison with other nonlinear measurements.

In Fig. 6 we compare our measurements for LDPE with measurements reported by Bastian (2001). The plot shows the Trouton ratio during a transient experiment at strain rates $\dot{\epsilon}=0.01 \mathrm{~s}^{-1}$ and $\dot{\epsilon}=1.0 \mathrm{~s}^{-1}$ as a function of Hencky strain. In the fast $\dot{\epsilon}=1.0 \mathrm{~s}^{-1}$ experiment, the RME measurements do not reach a plateau in elongational viscosity, but the measurements do seem to approach steady state. In our opinion the FSR shows a convincing plateau that is reached about half a strain unit later than in the RME. The plateau in the RME experiments is elevated about 25\% above the FSR measurements. 


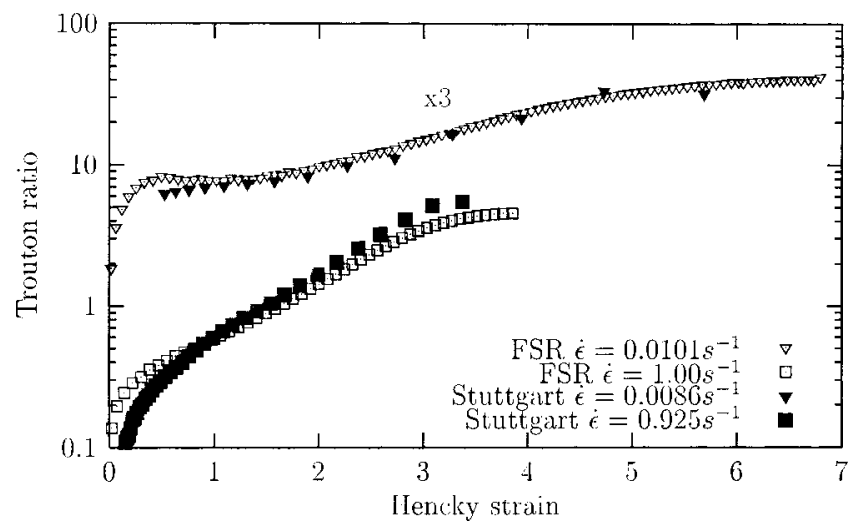

FIG. 6. Trouton ratio for LDPE $\left(150^{\circ} \mathrm{C}\right)$ as function of Hencky strain measured in the FRS compared with measurements in a RME by Bastian (2001). The FSR data are measured at initial aspect ratio $\Lambda_{0}=0.56$. The data for $\dot{\epsilon}=1.00$ and $0.925 \mathrm{~s}^{-1}$ are multiplied by 3 .

The slow experiments performed at $\dot{\boldsymbol{\epsilon}}=0.01 \mathrm{~s}^{-1}$ shown in Fig. 4 shows good agreement. Both the FSR and RME measurements reach high strains and a high plateau in Trouton ratio. The transients also agree well despite the $15 \%$ difference in strain rate.

We also compare measurements done on the LLDPE performed in the FSR with measurements reported by Schulze et al. (2001) in Fig. 7. The figure shows the Trouton ratio as function of Hencky strain for strain rates around $\dot{\epsilon}=0.1 \mathrm{~s}^{-1}$ and $\dot{\epsilon}=1.0 \mathrm{~s}^{-1}$ measured at the Minneapolis RME [(Schulze et al. (2001), Fig. 8)], on the prototype Meissner and Hostettler instrument in Zürich [(Schulze et al. (2001), Fig. 3)] and in our FSR.

We have a good agreement between the three sets of data in the range of Hencky strains $1-3$ especially at $\dot{\epsilon}=0.1 \mathrm{~s}^{-1}$. For $\dot{\epsilon}=1.0 \mathrm{~s}^{-1}$ the FSR data are delayed half a strain unit compared to the Minneapolis data but only about a quarter strain unit relative to the Zürich data. For larger Hencky strains the FSR reaches a steady state with a plateau

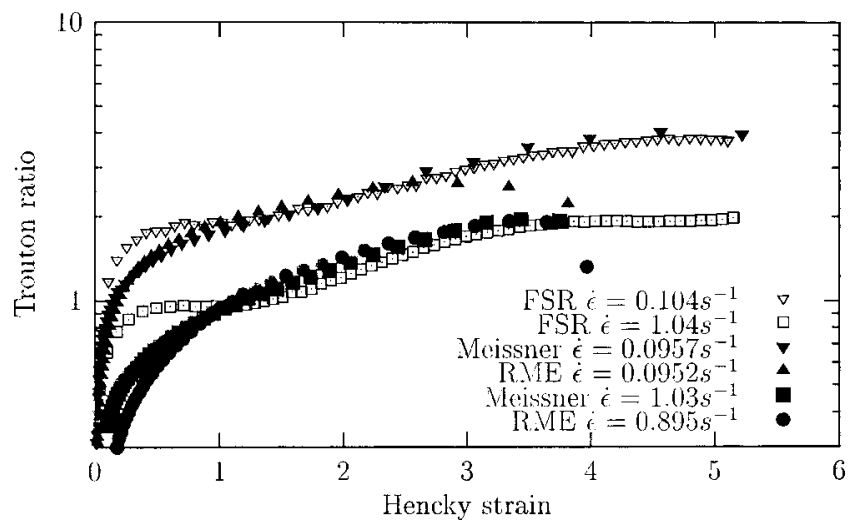

FIG. 7. Trouton ratio for LLDPE $\left(130^{\circ} \mathrm{C}\right)$ as function of Hencky strain measured in the FSR at strain rates $\dot{\epsilon}=0.10 \mathrm{~s}^{-1}(\nabla)$ and $\dot{\epsilon}=1.0 \mathrm{~s}^{-1}(\square)$ compared with RME measurements on a rheometer in Minneapolis and on the Meissner Hostettler prototype RME instrument in Zürich as reported in [Schulze et al. (2001)]. The FSR data are measured at initial aspect ratio $\Lambda_{0}=0.56$. 


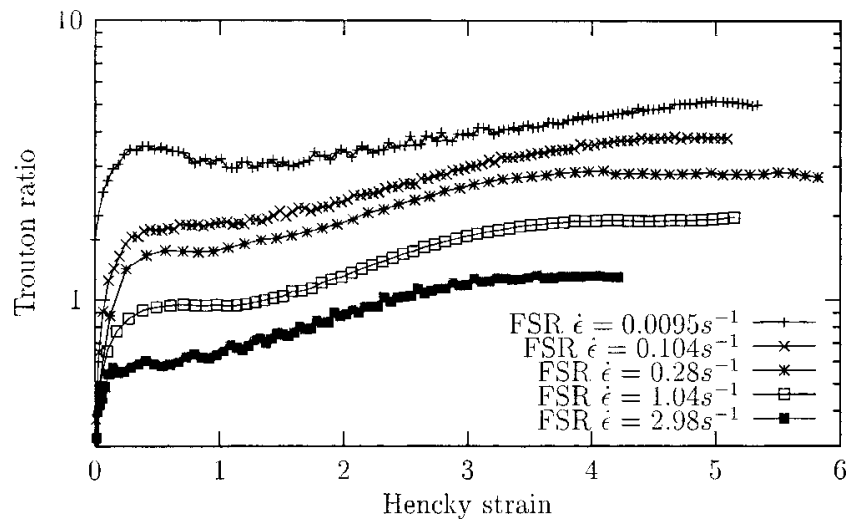

FIG. 8. Trouton ratio as of the LLDPE melt as function of Hencky strain during startup of elongational flow at five selected elongation rates measured in the FSR.

of nearly two Hencky strain units in good agreement with the Zürich data, while the Minneapolis instrument does not seem to reach steady state.

\section{E. LDPE and LLDPE}

Finally, we present our complete data series for LLDPE and LDPE in Figs. 8 and 9, respectively. Keep in mind, that our data should not be used for Hencky strains less than unity. We notice that steady state is obtained in all experiments albeit for a wider interval of Hencky strain with LLDPE than with LDPE. The instability leading to failure occurs much more rapidly for LDPE than for LLDPE. Hence, it is easier to adjust the plate motion for LLDPE than for LDPE. The strongly nonlinear elastic behavior of LDPE is also evidenced by the high Trouton ratios obtained for this material.

\section{CONCLUSION}

A filament stretching rheometer capable of measuring elongational viscosity at high temperatures has been constructed. At Hencky strains less than about unity, the transient elongational viscosity measured by the instrument is dependent upon the initial aspect ratio of the filament. By using a large initial aspect ratio, agreement is obtained with the

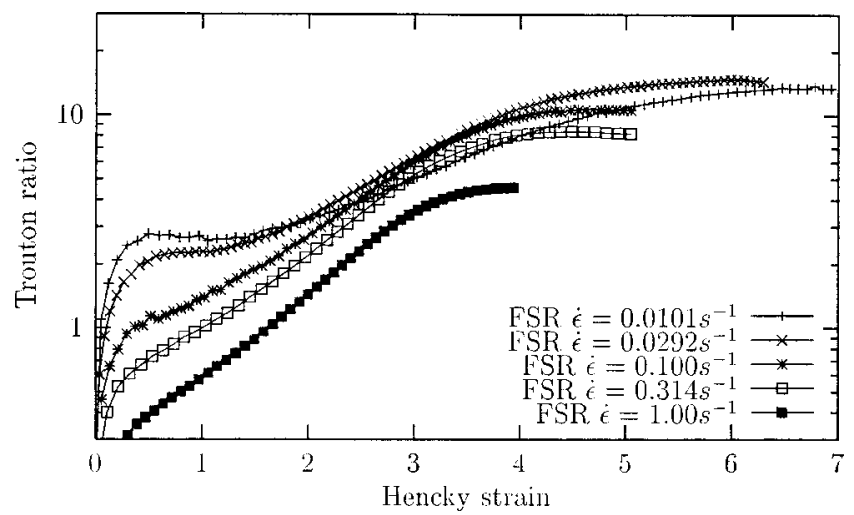

FIG. 9. Trouton ratio of the LDPE melt as function of Hencky strain during startup of elongational flow at five selected elongation rates measured in the FSR. 
predictions from linear viscoelasticity based on shear measurements. At Hencky strains above unity, the measurements are independent of the initial aspect ratio of the filament.

Two polymer melts were used in our investigations, a strongly strain hardening LDPE and a slightly strain hardening LLDPE. We have compared our measurements with measurements on the same polymer melts in other laboratories. Measurements on LDPE are compared with measurements on a commercial RME rheometer in Stuttgart. LLDPE measurements are compared with measurements on a commercial RME instrument in Minneapolis and on the prototype RME instrument located in Zürich. In view of the difficulties in measuring true elongational viscosities for polymer melts, the agreement between measurements done on the RME rheometers and the filament stretching rheometer is good for both polymer melts. For the LLDPE melt some variance is observed between the measurements at the three laboratories, the FSR measurements being closer the measurements on the original RME than to the commercial instrument.

It is difficult to obtain a steady state elongational viscosity for the very strain hardening LDPE melt. This is due to the very rapid necking of the filament for this highly elastic material. For the less strain hardening LLDPE melt, the FSR method is, however, able to measure stationary elongational viscosity for as much as two strain units - a feature that seems to be difficult to obtain in the RME.

An advantage in using the FSR for measuring elongational viscosity of polymer melts is the ability to measure on less viscous fluids than in the RME. Also the amount of sample used in one FSR experiment is less than in the RME by approximately a factor of 5. However, it is necessary to make at least three experiments in the FSR before the plate separation function is converged. Finally, we note that the FSR measures all parameters online. Only a small amount of postprocessing of the data is necessary to establishing the strain rate from the online measurements of the radius. In the RME, a measure of strain during an experiment usually has to be obtained by observing the filament from a video tape or similar [Schulze et al. (2001)].

\section{ACKNOWLEDGMENTS}

The authors would like to thank Professor Wagner and Dr. Bastian for providing the test samples used in this work and for measurements done in the RME. Also, they would like to thank Dr. Schulze and Professor Schweizer for making their LLDPE measurements available to them.

\section{References}

Anna, S. L., G. H. McKinley, D. A. Nguyen, T. Sridhar, S. J. Muller, J. Huang, and D. F. James, “An interlaboratory comparison of measurements from filament-stretching rheometers using common test fluids," J. Rheol. 45, 83-114 (2001).

Bach, A., H. K. Rasmussen, and O. Hassager, "Growth of nonaxisymmetric disturbances of the free surface in the filament stretching rheometer: Experiments and simulation," J. Non-Newtonian Fluid Mech. 108, 163186 (2003).

Bastian, H., "Non-linear viscoelasticity of linear and long-chain-branched polymer melts in shear and extensional flows," Ph.D. thesis, Institut für Kunststofftechnologie der Universität Stuttgart, 2001.

Berg, S., R. Kröger, and H. J. Rath, "Measurement of extensional viscosity by stretching large liquid bridges in microgravity," J. Non-Newtonian Fluid Mech. 55, 307-319 (1994).

Bhattacharjee, P. K., J. P. Oberhauser, G. H. McKinley, L. G. Leal, and T. Sridhar, "Extensional rheometry of entangled solutions," Macromolecules 35, 10131-10148 (2002).

Bird, R. B., R. C. Armstrong, and O. Hassager, Dynamics of Polymeric Liquids, Vol. 1: Fluid Mechanics, 2nd ed. (Wiley Interscience, New York, 1987). 
Kolte, M. I., H. K. Rasmussen, and O. Hassager, "Transient filament stretching rheometer II: Numerical simulation," Rheol. Acta 36, 285-302 (1997).

Kröger, S. Berg, A. Delgado, and H. J. Rath, "Stretching behaviour of large polymeric and Newtonian liquid bridges in plateau simulation," J. Non-Newtonian Fluid Mech. 45, 385-400 (1992).

Matta, J. E., and R. P. Tytus, "Liquid stretching using a falling cylinder," J. Non-Newtonian Fluid Mech. 35, 215-229 (1990).

McKinley, G. H., and O. Hassager, "The Considère condition and rapid stretching of linear and branched polymer melts," J. Rheol. 43, 1195-1212 (1999).

McKinley, G. H., and T. Sridhar, "Filament-stretching rheometry of complex fluids," Annu. Rev. Fluid Mech. 34, 375-415 (2002).

Meissner, J., and J. Hostettler, "A new elongational rheometer for polymer melts and other highly viscoelastic liquids," Rheol. Acta 33, 1-21 (1994).

Munstedt, H., "New universal extensional rheometer for polymer melts. Measurements on a polystyrene sample," J. Rheol. 23, 421-436 (1979).

Orr, N. V., and T. Sridhar, "Probing the dynamics of polymeric solutions in extensional flow using step strain rate experiments," J. Non-Newtonian Fluid Mech. 82, 203-232 (1999).

Rasmussen, H. K., and O. Hassager, "The role of surface tension on the elastic decohesion of polymeric filaments," J. Rheol. 45, 527-537 (2001).

Schulze, J. S., T. P. Lodge, C. W. Macosko, J. Hepperle, H. Münstedt, H. Bastian, D. Ferri, D. J. Groves, Y. H. Kim, M. Lyon, T. Schweizer, T. Virkler, E. Wassner, and W. Zoetelief, "A comparison of extensional viscosity measurements from various RME rheometers," Rheol. Acta 40, 457-466 (2001).

Solomon, M. J., and S. J. Muller, "The transient extensional behavior of polystyrene-based Boger fluids of varying solvent quality and molecular weight," J. Rheol. 40, 837-56 (1996).

Spiegelberg, S. H., D. C. Ables, and G. H. McKinley, "The role of end-effects on measurements of extensional viscosity in filament stretching rheometers," J. Non-Newtonian Fluid Mech. 64, 229-267 (1996).

Spiegelberg, S. H., and G. H. McKinley, "Stress relaxation and elastic decohesion of viscoelastic polymer solutions in extensional flow," J. Non-Newtonian Fluid Mech. 67, 49-76 (1996).

Sridhar, T., V. Tirtaadmadja, D. A. Nguyen, and R. K. Gupta, "Measurement of extensional viscosity of polymer-solutions," J. Non-Newtonian Fluid Mech. 40, 271-280 (1991)

Szabo, P., "Transient filament stretching rheometer part I: Force Balance analysis," Rheol. Acta 36, 277 (1997).

Van Nieuwkoop, J., and M. M. O. Muller Von Czernicki, "Elongation and subsequent relaxation measurements on dilute polyisobutylene solutions," J. Non-Newtonian Fluid Mech. 67, 105-23 (1996).

Verhoef, M. R. J., B. H. A. A. Van den Brule, and M. A. Hulsen, "On the modelling of a PIB/PB Boger fluid in extensional flow," J. Non-Newtonian Fluid Mech. 80, 155-82 (1999).

Wagner, M. H., H. Bastian, P. Hachmann, J. Meissner, H. Münstedt, S. Kurzbeck, and F. Langouche, "The strain hardening behaviour of linear and long-chain-branched polyolefin melts in extensional flows," Rheol. Acta 39, 97-109 (2000). 